\title{
Jóvenes rurales en la escuela secundaria del campo: una etnografía sobre estudiantes en el norte entrerriano
}

\author{
Emilia Schmuck
}

\begin{abstract}
Resumen
En el trabajo nos proponemos aportar a la comprensión de los procesos de educación y escolarización de las juventudes rurales en un contexto de extensión de la obligatoriedad escolar. A través de una investigación etnográfica que realizamos en una población rural del norte de la provincia de Entre Ríos, afirmamos que la escolarización de las y los jóvenes no es solo resultado del establecimiento de la obligatoriedad escolar por ley ni de la acción u omisión del estado en garantizarla, sino que se relaciona con el fuerte involucramiento de las familias y el lugar asignado a la educación en la reproducción generacional. Sin embargo, consideramos que esto no alcanza para explicar la relación entre las juventudes rurales y la escuela del campo, ya que éstas valoran fundamentalmente la escuela como posibilidad de encuentro con sus pares, como espacio de sociabilidad. La celebración del Día del Estudiante Rural, donde se construyen relaciones de afinidad que marcan una ruptura respecto a lo que sucede en el espacio social rural, nos permite avanzar en la comprensión de los modos en que las y los jóvenes se apropian, crean y recrean el espacio escolar.
\end{abstract}

Palabras clave: juventudes rurales, educación rural, educación secundaria, etnografía

\footnotetext{
* Licenciada en Comunicación Social por la Universidad Nacional de Entre Ríos (UNER). Becaria doctoral del Instituto de Estudios Sociales INES, UNER/CONICET. e-mail: emilia.schmuck@gmail.com
} 


\title{
Rural youth in secondary school in rural areas: an ethnography about students in the north entrerriano
}

\begin{abstract}
The work aims to contribute to the understanding of the transformations related to education and schooling of rural youth in a context of extension of the compulsory school. It refers to an unfinished research made according to the ethnographic approach in the north of the province of Entre Ríos (Argentina). We affirm that the schooling of young people it is not the result of the establishment of compulsory school by law or the action or omission of the state in guaranteeing it. Instead, we consider the importance of the involvement of local actors in the development of education and the place assigned to education in generational reproduction. However, we believe that this is not enough to explain the relationship between rural youths and the rural school since they fundamentally value the school as a possibility of meeting their peers, as a space of sociability. The celebration of the Rural Student's Day, where relationships change what happens in the rural social space, allows us to advance in the understanding of the ways in which young people appropriate, create and recreate the school space.
\end{abstract}

Keywords: rural youth, rural education, secondary school, ethnography 


\section{Introducción}

En este artículo nos proponemos aportar a la comprensión de los procesos de educación y escolarización de las juventudes rurales considerando cómo se configuran las prácticas y experiencias formativas de las y los jóvenes y sus familias en un contexto de extensión de la obligatoriedad escolar. A través de una investigación etnográfica en curso que realizamos en una población rural del norte de la provincia de Entre Ríos, que se caracteriza por su alejamiento y dificultades de acceso respecto a centros rurales y urbanos, nos preguntamos particularmente por los modos de apropiación y los procesos de identificación que se generan en torno a una festividad en particular: la celebración del Día del Estudiante Rural.

A pesar de la abundante producción académica relacionada con las juventudes y la educación secundaria, podemos señalar cierta vacancia de estudios que abordan sus particularidades en contextos rurales. Esto guarda estrecha relación con que, en primer lugar, aunque desde la década de 1980 se ha desarrollado en América Latina el campo de estudios sobre juventudes, las investigaciones sobre jóvenes rurales han sido relativamente escasas (Chaves, 2009; Kessler, 2007; Weishemer, 2013). En Argentina, se destaca la condición de "invisibilidad" de las y los jóvenes rurales (Schmuck, en prensa), aunque en los últimos años la situación ha comenzado a revertirse en el ámbito académico. Entre los trabajos disponibles, podemos señalar algunas investigaciones de alcance nacional que se propusieron describir la situación de las juventudes rurales en todo el país luego de las transformaciones ocurridas durante la década de 1990 (Caputo, 2002; Román, 2003; Román, 2011); otro grupo de trabajos producidos en fechas más recientes y que se caracterizan por avanzar en la descripción o comprensión de la situación de las juventudes rurales de determinadas regiones del país (Bruniard, Jabif \& Bresson, 2007; Calvo, Mariotti \& Ochoa., 2015; González \& Román, 2012) y una última serie de investigaciones que desde estudios de caso o en caso permite profundizar en las particularidades de una región o 
actividad productiva determinada y busca generar aportes para pensar las concepciones sobre ruralidades y juventudes (Barés, 2016; Bober \& Neiman, 2011; Roa, 2017; Ruiz Peyré, 2010).

En relación a los trabajos disponibles sobre educación secundaria, en primer lugar cabe señalar que, motivados por más de dos décadas de continuas políticas de extensión de la escolarización secundaria en el país y la región, en los últimos tiempos en Argentina se han llevado adelante numerosos y diversos estudios sobre la temática (Pinkasz, 2015) situados en contextos urbanos (Aienson, 2002; Di Napoli, 2016; Jacinto, 2006; Kravetz, 2012; Miranda, 2013; Terigi, 2008; Tiramonti, 2015). A propósito de la educación secundaria rural, podemos mencionar algunos trabajos preocupados por la implementación del nivel medio en contextos rurales que parten de información estadística escolar y son realizados desde la perspectiva de la evaluación de la calidad del sistema educativo (Caillods \& Jacinto, 2006; Cappellacci \& Ginocchio, 2009; González, Mamanis, Prudant \& Scarfó, 2015). Además, nos interesa destacar una línea de producciones que avanza hacia la comprensión de los procesos de educación y escolarización de las juventudes desde la mirada socio-antropológica y el enfoque etnográfico que compartimos (Ambrogi, Cragnolino \& Romero Acuña, en prensa; Hirsch, 2016; Ligorria, 2016; Padawer, Greco \& Rodríguez Celín, 2013; Padawer, Rodríguez Celín, Canciani, Greco \& Soto, 2015; Romero Acuña, 2017). Finalmente, en lo que refiere al problema que nos ocupa, podemos mencionar un estudio exploratorio reciente que analiza cómo se ha llevado a cabo la ampliación de la obligatoriedad escolar en los espacios rurales de la provincia de Entre Ríos y que realiza aportes significativos a la temática, señalando la necesidad de profundizar en las indagaciones para abordar las particularidades de las heterogéneas escuelas diseminadas en el territorio entrerriano (Mayer \& Vlasic, 2016).

A partir de los antecedentes señalados, realizamos el presente trabajo en diálogo con los estudios sobre antropología y educación desarrollados en América Latina, que se han llevado adelante durante los últimos 30 años en Argentina y, como expresa un trabajo que reconstruye sus principales aportes (Cerletti, 2017), se han 
consolidado en la última década. Nos referimos a una tradición de trabajos de investigadoras del país y la región (Achilli, 2005; BataIlán \& Neufeld, 1988; Ezpeleta \& Rockwell, 1983; Neufeld, 2014; Rockwell, 2009; Rockwell \& Mercado, 1986) que retoman aportes clásicos de la antropología y la historiografía, recuperando la dimensión cotidiana y experiencial de los sujetos desde un enfoque relacional, en tensión con las condiciones objetivas del contexto sociohistórico del que forman parte, considerando las limitaciones y posibilidades que el contexto imprime (Cerletti, 2013). En esta línea, entendemos que la etnografía, concebida en tanto proceso que a partir de la recuperación de lo cotidiano permite documentar lo no-documentado (Rockwell, 2009) tiene una potencialidad particular para estudiar la complejidad de las realidades rurales, las familias, la educación y la cotidianeidad escolar como parte central de esa vida social (Cragnolino, 2007a). La investigación en la que se enmarca este trabajo parte de la experiencia de participación en diferentes actividades familiares y sociales de una colonia del norte entrerriano y su escuela primaria y secundaria desde febrero de 2017. ${ }^{1}$ También hemos realizado entrevistas etnográficas (Pizarro, 2014) a ex directivas, docentes, padres y madres de estudiantes y hemos analizado fuentes documentales que se encuentran en el centro de documentación del Consejo General de Educación y el archivo de las escuelas.

Cabe señalar que el relato que incluimos está basado fundamentalmente en vivencias compartidas y entrevistas realizadas durante la preparación y los festejos del Día del Estudiante Rural en $2017^{1}$ y, principalmente, 2018. Aquí nos valemos del estudio etnográfico que Levinson (2002) realiza de los espacios, los rituales y las rutinas escolares en una escuela secundaria en México. El autor entiende que, creados en la interacción de las culturas estatal y local, los espacios, los rituales y las rutinas escolares entrelazan distintos elementos: cívicos, religiosos y de identidad juvenil; muestran cómo la historia se "convierte en estructura" (p.193) y

\footnotetext{
${ }^{1}$ De aquí en adelante, hablaremos de "la colonia" y "la escuela de la colonia" para preservar sus nombres.
} 
cómo los alumnos se apropiaban del espacio, el grupo y los discursos institucionales para producir sus estrategias, propios valores y formas culturales.

Este trabajo se organiza en cinco partes. En primer lugar, referimos a la historia de la población y la escuela en relación a los cambios en la provincia y la región. En el segundo apartado, remitimos al modo en que la condición de juventud se encuentra imbricada con la condición de estudiante en la colonia y mencionamos las particularidades de las y los diferentes jóvenes que asisten a la escuela. En tercer lugar, nos centramos en los procesos de identificación de las y los diferentes jóvenes en torno a la conformación de equipos deportivos para competir en la fiesta del Día del Estudiante Rural. Finalmente, desarrollamos algunas reflexiones finales y líneas para futuras indagaciones.

\section{Una escuela secundaria en "el lejano norte"}

El surgimiento de la población estudiada no se relaciona directamente con el proceso más referenciado por los trabajos sobre colonización en la provincia de Entre Ríos, que refieren al establecimiento de inmigrantes europeos a fines de siglo XIX (Bosch, 1973, 1978; Djenderedjian, 2006; Reula, 1969). En cambio, se vincula con las políticas de colonización tardía, que entre 1934 y 1987 significaron el asentamiento en los departamentos del centro y el norte entrerriano de la población que provenía fundamentalmente de otros puntos de la provincia y se dedicó a producciones como el algodón, la citricultura, el tambo, etc. (De Paoli, 1993; Mayer, 2014). Particularmente entre 1960 y 1965, se fundaron varias colonias en el norte del departamento La Paz, entre las que se encuentra este centro de población rural que surgió a partir de la compra estatal de tierras a un estanciero y su loteo en 52 unidades de entre 50 y 90 hectáreas. $^{2}$

\footnotetext{
${ }^{2}$ Escuela Secundaria Departamento La Paz (2014). Periódico Nuestro Lugar [Material de Archivo]. Secretaría de Agricultura Familiar de Entre Ríos, Entre Ríos. Es nuestra decisión de omitir la referencia precisa a la institución estudiada para preservar su intimidad.
} 
La historia de esta población se encuentra fuertemente asociada a la de su escuela primaria, ya que a los pocos años, en 1965, un grupo de colonos se autoconvocó,

con la iniciativa de formar una Comisión Cooperadora Pro Escuela Provincial (...). Establecidos todos los contactos imprescindibles para el logro de este anhelo e iniciados los trámites pertinentes, no quedaba más que esperar y rogar que fuera aprobada esta solicitud, considerada como una necesidad apremiante, por la cantidad de familias que se habían comenzado a establecer desde su colonización (...). Comenzó a funcionar el 5 de Setiembre de 1966, en un local cedido por el señor (...), hasta que el Consejo General de Educación designó la construcción de un edificio escolar en el terreno donado. ${ }^{3}$

Desde entonces, la escuela funciona en el espacio referenciado como el "centro de la colonia", conformado además por el Centro de Salud, el club, un almacén y un par de caseríos más, ubicados a $40 \mathrm{~km}$ de la ciudad más próxima y alrededor de $20 \mathrm{~km}$ de una vía asfaltada. Los recuerdos de las y los primeros pobladores con quienes aún es posible encontrarse, así como los relatos recuperados en documentos y proyectos escolares, dan cuenta de una colonia "granjera y ganadera" y "rodeada de monte nativo", muy diferente a la del presente:

La mitad de esa superficie estaba cubierta de monte y la otra mitad, de chacras (...). Los cultivos que se realizaban, y en orden de importancia de superficie sembrada, eran: lino, maíz, sorgo, granífero, algodón, avena, trigo y pradera permanente. ${ }^{4}$

Las épocas de prosperidad, que podemos situar hasta mediados de la década de 1980, también se relacionan con "la edad de oro del Club" fundado atrás del predio escolar y los logros deportivos en la Liga Departamental Paceña de Fútbol entre mediados de la década de 1970 y principios de 1980. Luego de haber permanecido muchos años en estado de abandono, en 2014 el club fue recuperado con fondos estatales a partir de gestiones de un técnico

\footnotetext{
${ }^{3}$ Escuela Primaria Departamento La Paz (2016). Discurso por Acto académico por los 50 años de historia [Material de Archivo]. Escuela Secundaria, Entre Ríos.

${ }^{4}$ Escuela Primaria Departamento La Paz (1983). La escuela. [Material de Archivo]. Escuela Primaria, Entre Ríos.
} 
de la entonces Secretaría de Agricultura Familiar ${ }^{5}$ y sus canchas actualmente se utilizan fundamentalmente para las prácticas de Educación Física de la escuela secundaria.

En correspondencia con las transformaciones en el sector agropecuario argentino vinculadas con reformas en la estructura y condiciones de producción, la desregulación de los mercados y la liberación de cultivos transgénicos a partir de la década de 1990, que a su vez significaron la profundización del avance de la agricultura en detrimento de otras producciones iniciada en la década de 1960 y 1970, la provincia de Entre Ríos atravesó una importante expansión de la frontera agrícola favorecida fundamentalmente por la introducción de la soja (Domínguez \& Orsini, 2009). De estas transformaciones no quedó exento el norte entrerriano, donde también podemos situar a los departamentos Feliciano y Federal, que junto al norte y este del departamento La Paz se caracterizan por su alto porcentaje de población rural, índices de analfabetismo que duplican la media provincial (Petitti, Chelotti \& Schmuck, 2018) y el predominio de suelos con limitaciones que restringen la elección de cultivos y abonan al imaginario de un "norte improductivo" (Schmuck, 2017). ${ }^{6}$

\footnotetext{
${ }^{5}$ Se trata de un trabajador con una experiencia de más de 10 años de trabajo en la zona, quien a comienzos de septiembre de 2018 ha sido apartado de su cargo en el marco de ola de despidos sustanciados desde la cartera de agroindustria, actualmente encabezada por Luis Miguel Etchevehere, productor entrerriano y ex Presidente de la Sociedad Rural Argentina (SRA). Estos despidos afectaron a 548 personas a nivel nacional, y entre ellas a 20 técnicos de la provincia de Entre Ríos, que se sumaron a los 16 despidos en el mes de abril en el contexto del proceso de vaciamiento de estos espacios y políticas estatales que se vienen advirtiendo desde el comienzo del gobierno de Cambiemos.

${ }^{6}$ Los índices de analfabetismo en los departamentos La Paz, Feliciano y Federal para el año 2010 duplican la media provincial y nacional de un $2 \%$ y en el caso de $\mathrm{Fe}-$ liciano llegan prácticamente al $5 \%$. Junto a Islas de Ibicuy son los distritos con mayor porcentaje de analfabetismo, seguidos, aunque en menor medida, por Federación, Gualeguay, Paraná, San Salvador, Victoria y Villaguay (con un 3\%). También en el norte, el promedio de utilización de computadoras en viviendas particulares es menor que la media provincial. Si tomamos otros indicadores como la utilización de suelos, el tipo de desagüe o el combustible para cocinar, la zona posee mayores condiciones desfavorables que la media provincial (Petitti et al., 2018).
} 
El proceso de decrecimiento y envejecimiento de la población rural -particularmente de la población rural dispersa- que marca el comportamiento demográfico de la provincia de Entre Ríos desde comienzos de la década de 1990 en relación con los cambios mencionados (Visintini \& Welsch, 2017), se evidencia fuertemente en la zona. Particularmente los informes producidos por trabajadores del Instituto Nacional de Tecnología Agropecuaria (INTA), sede La Paz, dan cuenta de cómo en las colonias del norte, el avance de la siembra directa ha acelerado la expulsión de pequeños y medianos productores y pobladores rurales dedicados al trabajo rural temporario (Dupleich, 2010). La colonia, de acuerdo al último censo, tiene una población de 396 habitantes considerando también los habitantes de la colonia vecina con la que integra una misma Junta de Gobierno (Instituto Nacional de Estadística y Censos [INDEC], 2010): "muchos fueron vendiendo...o regalando", "quedamos los menos", "antes había otro movimiento", son algunas de las expresiones que pueden escucharse cotidianamente entre las y los pobladores con más años.

Esta disminución de la población también se vislumbra en la evolución de la matrícula de la escuela. En la década de 1970, la escuela primaria llegó a tener 90 alumnos, cifra que comenzó a disminuir a fines de la década de 1980 hasta que a partir de 2006 la escuela pasó a ser de Personal Único. ${ }^{7}$ Sin embargo, podemos señalar que en 2000 se registra un aumento significativo en la matrícula por la creación de nuevos cursos que significaron el inicio del proceso de extensión de la escolaridad.

De acuerdo con la Ley Federal de Educación sancionada en 1993, a fines de 1997 en la provincia comenzó el proceso de extensión de la obligatoriedad escolar con la implementación de las primeras experiencias de creación del tercer ciclo de la Escolaridad General Básica (EBG) en escuelas rurales de la provincia (Doval, 2014). En 2006, se sancionó la Ley de Educación Nacional $N^{\circ} 26.206$ (LEN), que estableció la obligatoriedad de la escuela

\footnotetext{
${ }^{7}$ Escuela Primaria Departamento La Paz (2006). Distribución anual del personal directivo y docente [Material de Archivo]. Escuela Primaria, Entre Ríos.
} 
secundaria y un conjunto de modalidades educativas, entre las que se encuentra la educación rural; esta legislación tuvo su correlato en Entre Ríos en la Ley de Educación Provincial N 9.890, en 2008. A partir de entonces, en la provincia se crearon numerosas y heterogéneas escuelas secundarias en contexto rural, expansión que se concretó sobre la base de las escuelas primarias preexistentes y estuvo condicionada por la complejidad específica de las instituciones con dificultades de accesibilidad y baja densidad de población (Mayer \& Vlasic, 2016).

No obstante, identificamos cómo la preocupación por garantizar el acceso a la educación secundaria en el campo antecede a los cambios en la legislación y las iniciativas del Estado, ya que las familias se organizan para recaudar dinero y conformar grupos de trabajo para construir las aulas necesarias para la creación de nuevos cursos. De acuerdo a un documento producido para solicitar la creación del Polimodal por parte de la "comunidad educativa”, expresión que incluye a las familias, a quienes puntualmente integran la Asociación Cooperadora, a directivos y docentes de la zona y también provenientes de otras localidades, la extensión de la escolaridad en el campo se fundamenta ante la importancia de la "ampliación de la matrícula, brindar ofertas educativas completas y evitar el desarraigo familiar" y "la necesidad de nuestros alumnos de culminar sus estudios en la región" por "el desarraigo social y emocional de cada uno de ellos, el alejamiento de sus familias y amigos, el alto crecimiento de falta de esperanzas en un futuro mejor por la necesidad laboral de estas personas". ${ }^{8}$

En el año 2000, comienzan a crearse el tercer ciclo del EGB y desde 2006 la escuela, bajo la denominación de "Educación común de modalidad rural", ${ }^{9}$ garantiza la escolaridad secundaria

\footnotetext{
${ }^{8}$ Escuela Secundaria Departamento La Paz (2005). Proyecto para la Implementación del Polimodal [Material de Archivo] Escuela Secundaria, Entre Ríos.

${ }_{9}^{9}$ Aquí, nos parece importante señalar la diferencia entre las escuelas agrotécnicas, establecimientos de nivel medio cuyo objeto de estudio es "lo agropecuario", de las escuelas rurales o en contexto rural, instituciones que, como es el caso de la escuela que estudiamos, están ubicadas en el medio rural (Mayer \& Vlasic, 2016; Plencovich, Constantini \& Bocchiocchio, 2009). En la colonia, las escuelas
} 
completa. La generación de los padres y las madres de quienes actualmente son estudiantes de la institución se enorgullecen de contar con una escuela secundaria que permita que su descendencia "se quede" y no tenga que migrar para continuar sus estudios. Esta posibilidad, que aparece una y otra vez como una novedad que tranquiliza a las familias, suele subrayarse junto al relato de sufrimiento de las y los jóvenes que décadas atrás marcharon con 12 o 13 años a hacer la secundaria: vivir en pensiones o casas de familiares en la ciudad más cercana, con pocas o nulas posibilidades de volver a visitar a la familia por los problemas de acceso y la falta de recursos, partir a los internados de escuelas ubicadas en distintos puntos de la provincia y tener que aprender a convivir con muchas personas dejando las comodidades del hogar, entre otras narraciones marcadas por el sacrificio propio y de las familias para garantizar la finalización del nivel medio. En el caso de quienes, por imposibilidades económicas o distintos sucesos en sus trayectorias de vida como tener hijos o establecer una pareja con alguien de la zona, no terminaron la secundaria, la valoración aparece ligada al lamento por no haber tenido esa "suerte" o "posibilidad".

\section{Las juventudes rurales en la escuela: en cuatri, en traffic o en bici}

Así como los niños y las niñas asisten a la escuela primaria -o incluso inician su trayectoria escolar a partir del nivel inicial garantizado en algunas instituciones de la zona- y atraviesan sus

primarias de la zona y la secundaria no cuentan con una propuesta de formación agropecuaria o agrotécnica. La escuela secundaria inicialmente funcionó como Bachiller con orientación en Bienes y Servicios Agropecuarios y contó con asignaturas como "Conservación y Almacenamiento de Alimentos", "Producción vegetal", "Introducción a la informática aplicada a los procesos productivos" y "Mejorando la producción animal" y "Anatomía y Fisiología de nuestros animales domésticos." Escuela Secundaria Departamento La Paz, Entre Ríos (2006). Estructura curricular y modalidad orientada [Material de Archivo]. Escuela Secundaria, Entre Ríos. Luego la orientación fue reemplazada por Bachiller con Orientación en Economía y Administración de Empresas. Escuela Secundaria Departamento La Paz (2010). Plan de Estudio. Res. 3322/2010 [Material de Archivo]. Escuela Secundaria, Entre Ríos. 
infancias en el ámbito de la escuela, desde principios de siglo las y los jóvenes de la colonia asisten al secundario. La institución recibe a la gran mayoría de las y los egresados de las cuatro escuelas primarias rurales de la zona, por lo que la matrícula, que en 2018 llegó a 50 alumnos, está conformada por jóvenes que viven en un radio de hasta 12 kilómetros de distancia. ${ }^{10}$

El pasaje entre la primaria y la secundaria se encuentra, en la gran mayoría de los casos, garantizado: no hay dudas de que las y los egresados de las escuelas primarias continuarán sus estudios en la secundaria, aunque más adelante puedan repetir o abandonar. La escuela asegura el desayuno y almuerzo de las y los asistentes y existen becas de distinto orden que reciben un número significativo de estudiantes y se destinan en parte al pago de un transporte que traslada a quienes viven lejos. La deserción escolar, en este sentido, no es significativa en la zona y las bajas en la matrícula suelen explicarse a partir del pase de estudiantes a otra institución por el traslado de toda su familia: otra escuela rural en la región o una secundaria de las ciudades cercanas. ${ }^{11}$

En estrecha relación con otros trabajos que señalan la importancia que revisten las escuelas como espacios de sociabilidad entre pares en contextos rurales en Perú (Olivera Rodríguez, 2009) y para estudiantes de sectores urbano-populares y rurales en México (Ávalos Romero, 2012; Guerra \& Guerrero, 2004; Hernández, 2008; Weiss, 2015), visualizamos que para las y los estudiantes, la escuela aparece como el lugar en el que conocieron y se encuentran con sus amigos y amigas. La importancia de la sociabilidad (Weiss, 2015) en tanto disfrute por estar junto a otras y otros pares es valorada junto a la posibilidad única en ese contexto de "hacer algo": "vengo porque si no me aburro", "lo mejor es vernos y jugar al futbol o al vóley", "es venir o pasarme la mañana durmiendo". En lo relatos se destacan particularmente aquellas otras actividades

${ }^{10}$ Escuela Secundaria Departamento La Paz (2018). Planilla de asistencias [Material de Archivo]. Secretaría Escuela Secundaria, Entre Ríos.

${ }^{11}$ Escuela Secundaria Departamento La Paz (2000-2018). Libro de Actas de alumnos [Material de Archivo]. Secretaría Escuela Secundaria, Entre Ríos. 
que la institución genera a su alrededor promoviendo nuevas instancias de sociabilidad, como los campamentos, torneos deportivos, el gran festejo del Día del Estudiante y la recepción, entre otros momentos significativos en sus vidas.

Encontramos en las y los estudiantes una notoria disposición a recorrer numerosos kilómetros sobre caminos en malas condiciones para asistir cada mañana a la escuela y nos sorprende el buen ánimo que reina durante las largas esperas, que a veces duran hasta tres horas, que protagonizan todos los mediodías las y los jóvenes que retornan a su casa en el segundo viaje que realiza la única traffic de la zona. Esto implica que otras y otros jóvenes que viven más cerca y/o pueden decidir cuándo partir en su bicicleta, moto o auto, a veces demoren su regreso y se queden jugando a las cartas, charlando o compartiendo la señal de Internet de la institución.

Las y los jóvenes pasan de hecho muchas horas en la escuela. Esto explica por qué, ante la pregunta de una recién llegada acerca de cuántos jóvenes hay en la colonia, las y los lugareños responden refiriendo a la cantidad de estudiantes en la escuela sin pensarlo demasiado. Hay excepciones: algunos también refieren a los pocos jóvenes varones que ya han egresado y aparecen como jóvenes porque siguen viviendo en el campo y no han "hecho familia", es decir, no tienen pareja con la que viven y/o hijos. También se señala, siempre desde la preocupación o la pena porque "no han aprovechado la oportunidad", a otros pocos jóvenes que no han terminado los estudios y "han quedado en el campo". Estos jóvenes, aunque ya no asistan a clases, sin embargo se encuentran presentes en los eventos que suceden en la escuela, entran y salen del predio escolar como si estuvieran en sus casas, por lo que el espacio escolar también es central en su cotidianeidad. Lo mismo sucede con jóvenes que han egresado y viven en la ciudad cercana u otras localidades donde estudian y/o trabajan: aunque por el momento no vivan en el campo, no se permiten faltar a los eventos importantes y aunque por diversos motivos no puedan estar presentes, están al tanto de los preparativos y contingencias vinculadas a la fiestas de egresados o las competencias con otras escuelas. 
Aunque esta pertenencia es significativa para todas y todos, es importante señalar que alrededor de la institución, que como mencionamos se caracteriza por su alejamiento y dificultades de acceso respecto a centros rurales y urbanos, se produce el encuentro entre jóvenes que revisten diferentes condiciones económicas y sociales. No es posible entonces estudiar a las juventudes en abstracto, sino que es necesario contemplar su carácter dinámico y plural y la existencia de diferentes y desiguales modos de ser joven (Chaves, 2009; Reguillo, 2000; Saintout, 2009).

Estas desigualdades pueden advertirse particularmente en los momentos de ingreso y salida de la escuela: mientras algunos y algunas llegan en bicicleta y la mayoría realiza eternos viajes en la traffic, hay otros y otras que se trasladan en vehículos costosos, como es el caso de una joven que llega en un cuatriciclo y otro que maneja una camioneta muy llamativa y la estaciona al lado de los vehículos de las y los docentes. Las desigualdades económicas de sus familias también se evidencian al visitar sus viviendas, casas amplias con comodidades contrastan con modestas construcciones con pocos dormitorios y muchos habitantes. Al indagar acerca de las actividades que realizan por la tarde, cuando no están en la escuela, la diferencia suele radicar en que solo algunos y algunas tienen posibilidades de practicar algún deporte o disciplina en la ciudad, generalmente fútbol, en el caso de los varones, y jockey, en el caso de las mujeres. En cuanto a su relación con el trabajo, aunque no necesariamente haya un reconocimiento explícito del tiempo destinado a estas tareas, algunas jóvenes mujeres realizan actividades de cuidado dentro del hogar, mientras encontramos varones que trabajan con sus familias en los campos, generalmente realizando tareas más o menos esporádicas que en ningún caso interfieren con la asistencia a la escuela. En términos generales, el hecho de colaborar o no con el trabajo doméstico y productivo no se vincula con las condiciones materiales de la familia de cada joven, de hecho, encontramos en los jóvenes de las familia "más acomodadas" mayor tendencia a participar del trabajo familiar en los campos. Sin embargo, identificamos que solo los jóvenes con necesidades económicas 


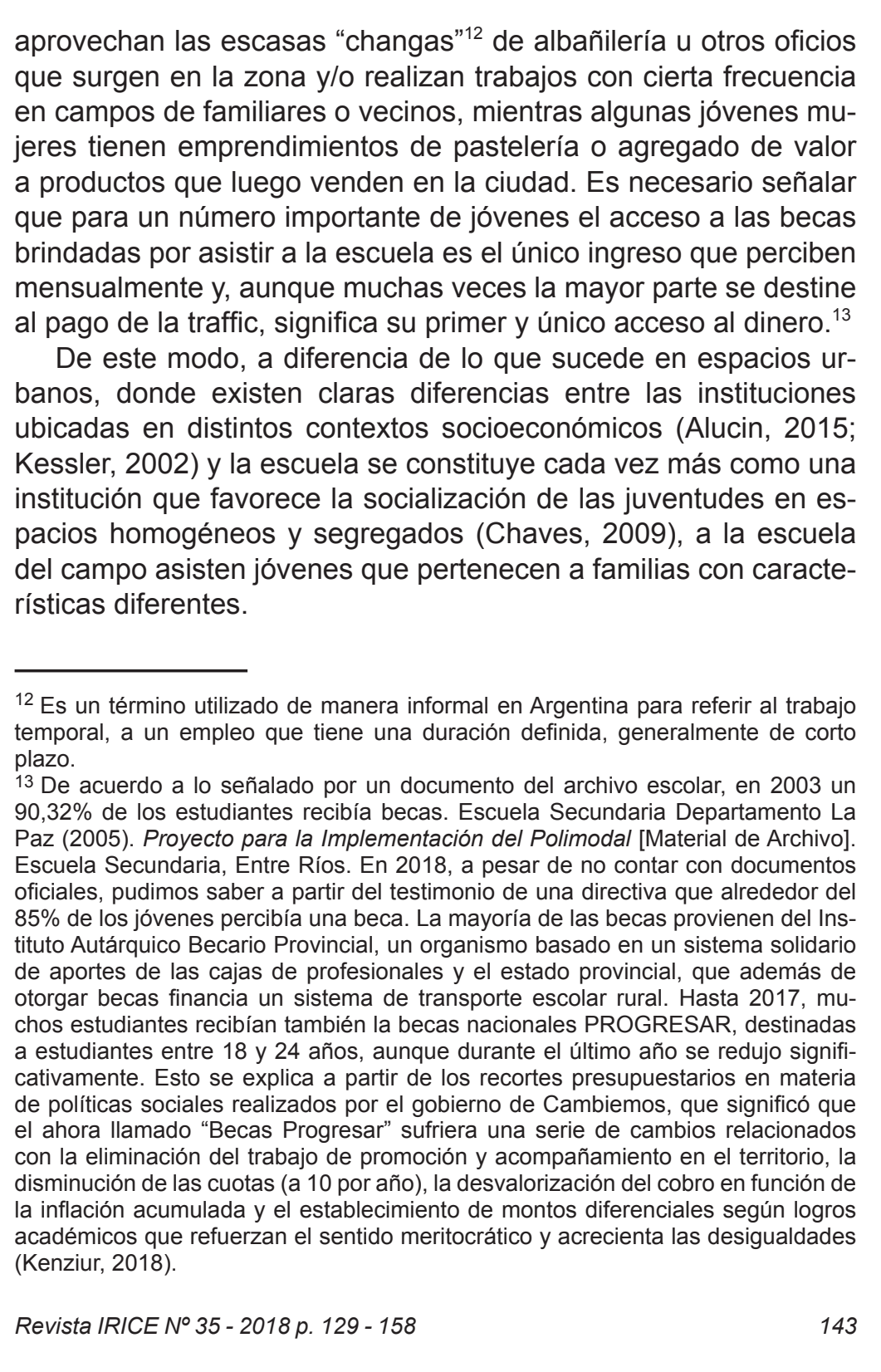


Las diferentes pertenencias de las y los jóvenes se relacionan con la existencia de dos grupos de familias que protagonizan tensiones en la cotidianeidad escolar. "La diferencia es entre los choriplaneros, o negros, y los gringos, que seríamos nosotros", determina una egresada de la escuela desde una mirada crítica hacia las diferencias en su lugar de origen. En palabras de la directora de la secundaria, estos dos grupos son "como si fueran los de la civilización y la barbarie". Con civilización se relaciona a las familias gringas, que "tienen más campo, gastan menos, tienen más recursos y por ahí por eso se creen que pueden opinar más y quieren decidir todo", mientras la barbarie se relaciona con aquellos que "se quedaron con poco campo, arriendan o son peones o cobran los planes, que por ahí no tienen tanto la cultura del trabajo, lo que le molesta a los otros".

La distinción también aparece relacionada con la zona en la que se encuentra la vivienda. Es en la región aledaña a la escuela, el centro de salud y el club donde suelen vivir las familias más distinguidas, con disputas deportivas originadas en las primeras décadas de la colonia y desengaños amorosos que explican peleas históricas entre determinados apellidos, aunque las diferencias materiales enraizadas en la ascendencia europea o criolla permite explicar las divisiones más tajantes entre los dos grupos. Cabe destacar que en esta diferenciación estamos excluyendo a los grandes propietarios que han logrado acaparar la mayor cantidad de hectáreas en los últimos años, quienes en el Proyecto Educativo Institucional de la Escuela son mencionados como "grandes productores con capitales no genuinos de la zona". ${ }^{14}$ Aunque varios estudiantes y docentes pueden mencionar algunos de sus apellidos, estos grandes productores que no viven en la zona ni envían a sus hijos a la escuela no tienen presencia en la vida escolar.

Cragnolino (2007a) identifica cómo a la creación de las escuelas en el norte cordobés la protagonizan grupos de "vecinos

\footnotetext{
${ }^{14}$ Escuela Secundaria Departamento La Paz (2006). Proyecto Educativo Institucional, versión 2006 [Material de Archivo]. Escuela Secundaria, Entre Ríos. 
distinguidos", que suelen ser los propietarios de los campos más extensos con acceso a las autoridades ministeriales y luego forman parte de la Cooperadora, ocupada por varones de posición social de importancia que se suceden por generaciones en los distintos cargos. En este sentido, en los documentos de la escuela primaria, los apellidos de dos o tres familias aparecen como quienes "generosamente" han donado los campos donde "con ayuda de todos" se construyeron las aulas; luego, estos mismos apellidos se suceden como presidentes y tesoreros de la cooperadora a lo largo de los años. Más allá de los conocimientos y la disposición para invertir tiempo y dinero, a falta de otras instituciones donde pueda darse esta disputa, se evidencia que la centralidad de estos espacios reside en que se constituyen como los únicos donde "obtener o incrementar el prestigio social” (Cragnolino, 2007b, p. 29).

Podemos señalar que dado que la escuela secundaria recibe a los egresados de todas las escuelas primarias de la región, significa la inclusión de familias provenientes de lo que es conocido como "la zona del arroyo" y la colonia aledaña, donde residen mayormente las familias que nuestros entrevistados de ascendencia europea han identificado como "criollos". Esta profundización de las heterogeneidades del alumnado -que no obstante se identifica desde los inicios de la escuela primaria- se advierte recién en los documentos escolares, que expresan que "la mayoría de los estudiantes provienen de familias con escasos recursos financieros. Un $80 \%$ son familias que se dedican a trabajos rurales donde el padre es empleado del dueño de varias hectáreas. Poseen planes sociales". ${ }^{15}$

Los distintos grupos de familias se encuentran en las reuniones de padres y madres y también en el marco de la asociación cooperadora de la secundaria creada en 2008. Aquí, a pesar de que el presidente pertenece a las familias que históricamente han ocupado esos espacios, también hay representación de nuevas familias, como puede advertirse en los apellidos que conforman la

\footnotetext{
${ }^{15}$ Escuela Secundaria Departamento La Paz (2011). Proyecto Educativo Institucional, versión 2011 [Material de Archivo]. Escuela Secundaria, Entre Ríos.
} 
asociación y distintos relatos sobre las discusiones. Los enfrentamientos entre los grupos son muy comentados en la escuela, por las propias familias y sobre todo por docentes y directivos. Una joven docente y egresada de la escuela sostiene que: "hay algunos que por ahí se animan a opinar, a dar su punto de vista, y si los que por ahí son más pizpiretas dijeron lo contrario, ya se quedaron callados". Entre los motivos de discusión mencionados por los distintos participantes en el último año, identificamos peleas por el destino de los fondos recaudados y diferencias sobre formas de proceder en eventos escolares y fiestas, aunque, en palabras de otra docente entrevistada "se pelean por enemistades de años, no importa cuál es el asunto pero ellos se pelean”.

\section{El equipo deportivo: todos y todas contra uno}

Hasta el momento, hemos referido a las diferencias y desigualdades entre las y los jóvenes y sus familias en la escuela secundaria. Sin embargo, nos interesa señalar que estas desigualdades suelen pasar inadvertidas en la cotidianeidad escolar, particularmente en espacios educativos marcados por los procesos de apropiación por parte de las y los estudiantes, quienes generan lazos identitarios al tiempo que buscan diferenciarse de otras escuelas.

Estos procesos se ponen en escena en su máxima expresión durante el festejo del Día del Estudiante Rural. La celebración emerge como un momento extraordinario donde las y los jóvenes son "los que copan la escuela", tomando la expresión de una estudiante. Desde 2001, el 21 de septiembre, cuando en Argentina se celebra el día del estudiante y la primavera, siete escuelas secundarias rurales de los departamentos La Paz y Feliciano organizan una jornada deportiva y cultural que se realiza alternadamente en las diferentes instituciones. "Hay torneo de vóley y fútbol y otras competencias que cambian según lo que decide la escuela que organiza, pueden ser ping pong de preguntas y respuestas, maquetas, baile, de sketch, después está la elección del rey y la reina, es un día entero", explica un joven ante la pregunta. Somos testigos del entusiasmo de distintos grupos de jóvenes durante los días 


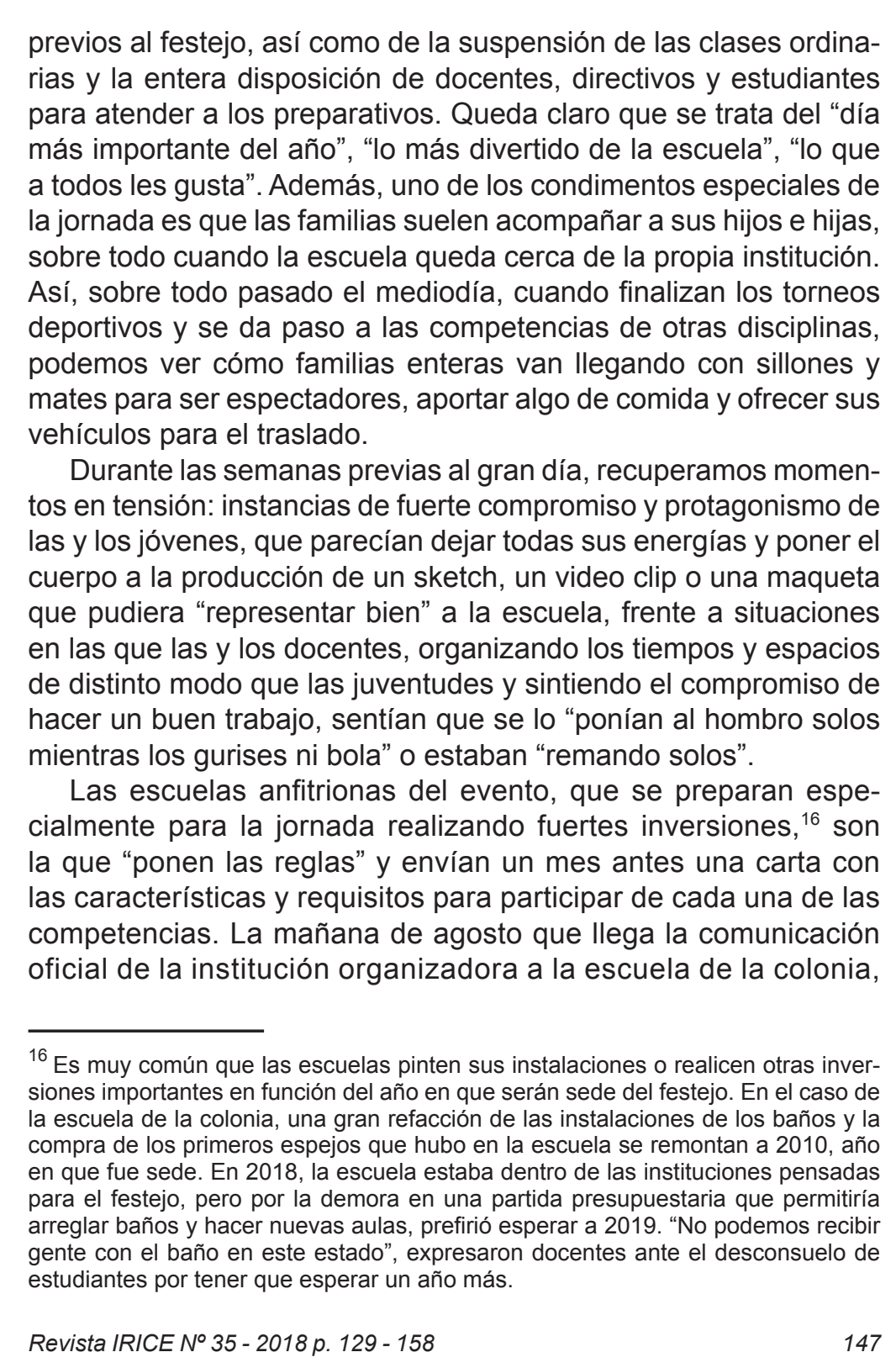


las y los estudiantes se convocan en el aula más grande de la escuela con el objetivo de leerla con atención y distribuir los equipos encargados de cada tarea. La principal tarea del centro de estudiantes a lo largo del año escolar tiene que ver con ordenar este proceso: la joven presidenta, estudiante de 4to año y con mucha habilidad para ser escuchada por sus compañeros y compañeras, eleva la voz para pedir silencio y atención. "No podemos perder el tiempo gurises, yo ahora voy a leer primero todo junto y después vamos parando en cada punto para armar los grupos", afirma con seguridad mientras el resto se organiza espontáneamente en semicírculo mirando al pizarrón y el escritorio de las y los docentes que ahora ocupa la presidenta. No hay presencia de adultos en ese espacio y cuando más tarde ingresan unos docentes a pedirles que se apuren porque es el horario del comedor y tienen que comunicarle unas cosas, se genera desorden en la circulación de la palabra que los representantes del centro de estudiantes estaban garantizando con su autoridad.

Al lado de la presidenta está el vicepresidente, quien en la votación del año anterior salió segundo y entonces asumió ese lugar. Juntos parecen constituir un buen equipo, aunque el joven se limita a anotar en el pizarrón y, a pesar de su alto perfil en la escuela -luego es elegido como candidato a rey de la primavera-, se mantiene en segundo plano mientras su compañera protagoniza la escena. La presidenta pertenece a una familia numerosa que vive en la zona identificada como de los "criollos", con disputas históricas con otras familias más "potentadas" en función de las diferencias que hemos señalado anteriormente. Sus padres conforman la cooperadora de la escuela primaria ubicada cerca de su casa pero no han tenido injerencia en la escuela de la colonia. El vicepresidente del centro de estudiantes, por su parte, es hijo del presidente de la cooperadora e integrante de una de las familias con más prestigio en la institución y recursos económicos de la zona. Existen relaciones laborales entre estas familias debido a que la familia del joven vicepresidente es "patrón" de una de las integrantes de la familia de la joven presidenta; sin embargo, en la escuela esa jerarquía no solo pasa desapercibida, sino que podemos decir que se invierte. 


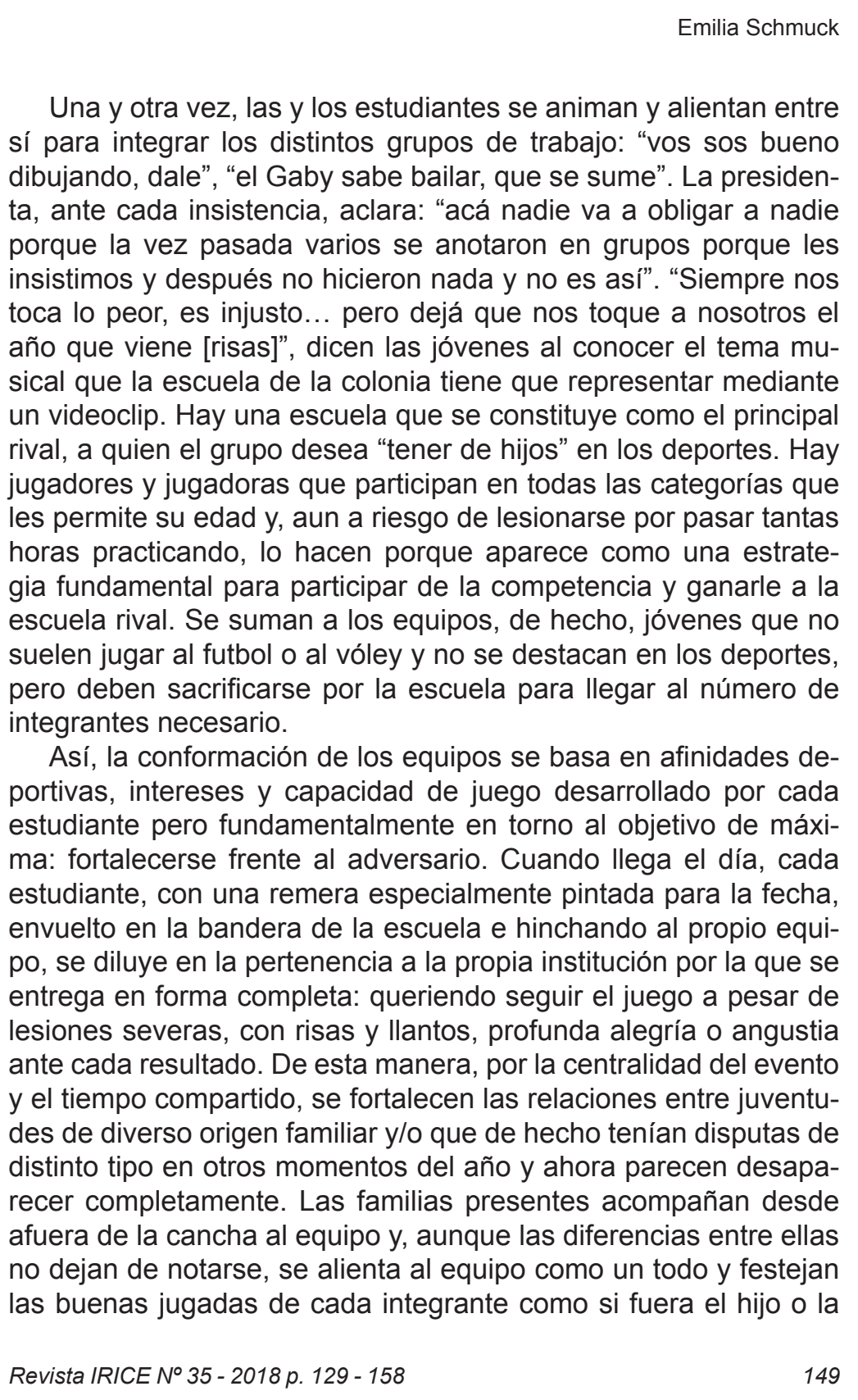


hija propia. De este modo, en relación a lo que expresan los estudios sobre culturas juveniles, que destacan la importancia de la producción de un otro antagónico que fundamenta la constitución de un "nosotros" (Reguillo, 2000), entendemos que en gran parte las identidades de estas juventudes se producen a partir de la diferenciación con los de afuera. Sin embargo, en el juego de las identidades, ante todo prevalece la construcción del otro semejante (Hernández, 2008), con quien se deja todo en la cancha, de igual a igual y hasta el final.

\section{Reflexiones finales}

A lo largo de este trabajo, pudimos comprender cómo en la colonia, desde la fundación de la escuela primaria por parte de un grupo de colonos en 1966, la presencia del "estado educador" estuvo condicionada por la participación de las familias. De este modo, la escolarización de las y los jóvenes rurales no es solo resultado del establecimiento de la obligatoriedad escolar por ley ni de la acción u omisión del estado en garantizarla, sino que fundamentalmente se relaciona con el fuerte involucramiento de las familias y el lugar asignado a la educación en la reproducción generacional.

En primer lugar, la educación y la escuela secundaria existe y es posible en el campo porque aparece como significativa en las estrategias de reproducción social de las familias (Cragnolino, 2001) y, además de constituirse como un atributo requerido por el sistema productivo, es reivindicada como mecanismo para superar la explotación y transformar la trama de relaciones sociales (Ezpeleta \& Rockwell, 1983). Además de valorar la "suerte" y la "posibilidad" de contar con la institución en la zona, las familias, según su posición social y económica, entienden la escuela secundaria como medio para superar la condición social, obtener un certificado que permita conseguir mejores empleos en la ciudad, continuar los estudios superiores y/o fortalecer el status de la persona o la familia. Podemos afirmar que la importancia de la escuela adquiere mayor peso en el caso de las jóvenes mujeres, 
quienes son interpeladas fundamentalmente por sus madres para continuar los estudios secundarios y, si es posible, terciarios o universitarios, y alentadas por docentes y directivas que insisten en evitar que las jóvenes queden embarazadas y abandonen sus objetivos de formación para ser madres. Esto puede relacionarse en parte con lo que sostienen los estudios realizados en el norte de Córdoba, que señalan que las familias de origen campesino asignan preferencia a la continuidad educativa de las mujeres porque tienen menos posibilidades objetivas de insertarse en el mercado de trabajo local (Cragnolino, 2001), pero también con los deseos por parte de las madres de que sus hijas concreten los anhelos que no pudieron cumplir y algunas imposiciones de género de la religión evangélica valdense que prima en la colonia en la que es necesario profundizar.

Sin embargo, consideramos que lo expresado no alcanza para explicar la relación entre las juventudes rurales y la escuela del campo. Las y los jóvenes valoran la escuela como posibilidad de encuentro con sus pares, como un lugar donde pasar el tiempo y divertirse. En este sentido, necesitamos pensar la escuela no solo como un espacio de socialización sino fundamentalmente de sociabilidad y avanzar en la comprensión de las particularidades que adquiere en un contexto rural marcado por la disminución de la población, la falta de otros espacios de encuentro y cierto aislamiento por las dificultades acceso a la escuela y la colonia. Si las y los jóvenes de las generaciones anteriores se encontraban en el club y ponían a jugar sus pertenencias e identificaciones en torno a los eventos sociales y deportivos que allí ocurrían, ahora la escuela, partícipe de la recuperación de la institución, es la única usuaria de sus instalaciones.

En la escuela de la colonia, además, se produce el encuentro con otras y otros jóvenes que pertenecen a diferentes y desiguales familias. A pesar de que no se transformen las relaciones sociales por fuera de la escuela, entendemos que los lazos identitarios que se construyen entre estudiantes, fundamentalmente alrededor de la competencia en el marco del festejo del Día del Estudiante Rural, permiten construir relaciones de horizontalidad y afinidad entre 
jóvenes que marcan una ruptura respecto a lo que sucede en el espacio social de la colonia. Si se entiende la apropiación como un proceso múltiple, relacional, transformador y arraigado en luchas sociales (Chartier, 1994; Rockwell, 2005), podremos ahondar en las apropiaciones selectivas y heterogéneas de las y los jóvenes, así como en los modos en que directivos, docentes y familias se apropian de los distintos "espacios, los tiempos, las palabras y los saberes en la escuela" (Rockwell, 2006, p. 7).

A partir de estudiar el proceso de disminución de la población en contextos rurales y en la colonia, afirmamos que muchas de las y los egresados de la escuela secundaria continúan sus trayectorias de vida en otro lugar, aunque, a diferencia de la generación que los antecede, postergan la partida: se quedan en la escuela. Si bien la extensión de la escolaridad no necesariamente fomenta el arraigo ni frena la migración a la ciudad, prolonga la estadía y con ello la posibilidad de "ser joven rural". Ahora bien, la educación y la escuela secundaria no se limitan a constituirse como una "sala de espera vacía", sino que las juventudes rurales se apropian, crean y recrean el espacio escolar.

\section{Referencias}

Achilli, E. (2005). Investigar en Antropología Social. Los desafíos de transmitir un oficio. Rosario: Laborde.

Aisenson, D. (2002). Después de la escuela: transición, construcción de proyectos, trayectorias e identidad de los jóvenes. Buenos Aires: Eudeba.

Alucin, S. (2015). Políticas educativas para el nivel secundario un análisis antropológico centrado en las perspectivas de los docentes. Revista IRICE, 29, 99-128.

Ambrogi, S., Cragnolino, E. \& Romero Acuña, M. (2019). La obligatoriedad de la escuela secundaria en contextos rurales de Argentina: Desde las prescripciones normativas a las construcciones cotidianas en tres experiencias educativas. Acciones e investigaciones sociales de la Universidad de Zaragoza, 39, 133-157.

Ávalos Romero, J. (2012). Las conversaciones juveniles en los intersticios del aula. En E. Weiss (Ed.), Jóvenes y bachillerato (pp. 97-124). México: Asociación Nacional de Universidades e Instituciones de Educación Superior. 
Barés, A. (2016). Comunicación, movilidades y espacialidades. Desplazamientos y trayectorias de jóvenes de Ñorquin Co y Cushamen en la región de la Patagonia argentina. Revista Latinoamericana de Estudios Rurales, $1(1), 105-134$.

Batallán, G. \& Neufeld, M. R. (1988). Problemas de la antropología y la investigación educacional en América Latina. Cuadernos de Antropología Social, 2,1-6.

Bober, G. \& Neiman, M. (2011). Los jóvenes trabajadores agrícolas en el Valle de Uco, Mendoza, Argentina. Trabajo y Sociedad, 16(15), 85103.

Bosch, B. (1973). La colonización de Entre Ríos. El país de los argentinos. Buenos Aires: Centro Editorial de América Latina.

Bosch, B. (1978). Historia de Entre Ríos 1520-1969. Buenos Aires: Plus Ultra.

Bruniard, R., Jabif, L. \& Bresson, A (2007). Representaciones y demandas de jóvenes rurales del NOA y NEA argentino sobre sus oportunidades de desarrollo. En R. Bruniard (Ed.), La educación de los jóvenes de provincias del NEA y NOA en Argentina (pp. 68-121). Recuperado de http://unesdoc.unesco.org/images/0015/001507/150789s.pdf

Caillods, F. \& Jacinto, C (2006). Mejorar la equidad en la educación básica. Lecciones de programas recientes en América Latina. Buenos Aires: IIPE-UNESCO.

Calvo, C., Mariotti, D. \& Ochoa, N. (2015). La situación de la juventud rural en torno al acceso y permanencia en la tierra. Buenos Aires: Dirección Nacional de Juventud Rural. Secretaria de Coordinación Político Institucional y Emergencia Agropecuaria.

Cappellacci, I. \& Ginocchio, M. V. (2009). La educación secundaria rural en la actualidad. Boletín Temas de Educación, 7(4), 1-48.

Caputo, L. (2002). Informe de Situación. Juventud Rural Argentina 2000. Buenos Aires: Ministerio de Desarrollo Social y Medio Ambiente, Dirección Nacional de la Juventud.

Cerletti, L. (2013). Enfoque etnográfico y formación docente: aportes para el trabajo de enseñanza. Pro-Posições, 24(2), 81-93.

Cerletti, L. (2017). Antropología y Educación en Argentina: de condiciones de posibilidad, preocupaciones en común y nuevas apuestas. Horizontes Antropológicos, 23(49), 123-148.

Chartier, R. (1994). Cultura popular: retorno a un concepto historiográfico. Manuscrits Revista d'història moderna, 12, 43-62.

Chaves, M. (2009). Estudios sobre juventudes en Argentina. Hacia un estado del arte/2007. La Plata: Editorial de la Universidad Nacional de La Plata. 
Cragnolino, E. (2001). Educación y Estrategias de Reproducción Social en familias de origen campesino del norte de Córdoba (Tesis doctoral). Facultad de Filosofía y Letras de la Universidad de Buenos Aires, Buenos Aires.

Cragnolino, E. (2007a). Introducción. En Autor (Ed.), Educación en los espacios sociales rurales (pp. 4-11). Córdoba: Facultad de Filosofía y Humanidades, Universidad Nacional de Córdoba.

Cragnolino, E. (2007b). Esa escuela es nuestra: relaciones y apropiaciones de la escuela primaria por parte de familias rurales. En Autor (Ed.), Educación en los espacios sociales rurales (pp. 12-40). Córdoba: Facultad de Filosofía y Humanidades, Universidad Nacional de Córdoba.

De Paoli, G. (1993). Ampty Oyenden. Nuestra memoria. Historia de Entre Ríos. Paraná: Ediciones del Ateneo de Paraná.

Di Napoli, P. (2016). La violencia en las escuelas secundarias desde tres perspectivas de análisis. Hacia un estado del arte. Zona Próxima, 24, 61-84.

Djenderedjian, J. (2006). En la periferia de la pampa gringa. Algunas reflexiones en torno al proceso de expansión agrícola y colonización en Entre Ríos, 1850-1890. Trabajo presentado en las XX Jornadas de Historia Económica de la Universidad Nacional de Mar del Plata, Mar del Plata.

Domínguez, N \& Orsini, G. (2009). Impactos de la estructura agraria por la ampliación de la frontera agrícola en base a la expansión del cultivo de soja en la región Pampeana: la historia reciente en Entre Ríos. Buenos Aires: Ediciones Cooperativas.

Doval, D. (2014). Código disciplinar de Formación ética y ciudadana y manuales escolares homónimos para EGB3: relato(s) de ciudadanía(s). Entre Ríos, Argentina 1993-2003. En A. Civera, C. Escalante \& E. Rockwell (Eds.), Sujetos, poder y disputas por la educación. México: Universidad Pedagógica Nacional, El Colegio Mexiquense, Cinvestav.

Dupleich, J. (2010). El Desarrollo Territorial. Una experiencia en las colonias del norte del departamento. La Paz: INTA, AER.

Ezpeleta, J. \& Rockwell, E. (1983). Escuela y clases subalternas. Cuadernos Políticos, 37, 70-80.

González, C. \& Román, M. (2012). Juventud y migraciones: vivencias, percepciones, ilusiones: un estudio en NOA y NEA. Buenos Aires: Ministerio de Agricultura, Ganadería y Pesca.

González, D., Mamanis, S., Prudant, E. \& Scarfó, G. (2015). Panorama de la Educación Rural en Argentina. Boletín Temas de Educación, 12(15), 3-48. Recuperado de http://portales.educacion.gov.ar/wp-content/blogs.dir/37/files/2015/12/Boletin-12-1_12_15.pdf 
Guerra, I. \& Guerreno, E. (2004). ¿Qué sentido tiene el bachillerato? Una visión desde los jóvenes. México: Universidad Pedagógica Nacional.

Hernández, J. (2008). El trabajo de los estudiantes de bachillerato: reflexividad, voces y marcos morales. México: Universidad Pedagógica Nacional.

Hirsch, M. (2016). Construyendo futuro en contextos de desigualdad social: Una etnografía en torno a las elecciones de los jóvenes en la finalización de la escuela secundaria (Tesis doctoral inédita). Facultad de Filosofía y Letras de la Universidad de Buenos Aires, Buenos Aires.

Instituto Nacional de Estadística y Censos (2010). Censo 2010. Recuperado de http://www.indec.gov.ar/nivel4_default.asp?id tema_1=2\&id tema_2=41\&id_tema_3=135

Jacinto, C (2006). La escuela media: reflexiones sobre la agenda de inclusión con calidad. Buenos Aires: Santillana.

Kenziur, M. (2018). Reconstrucción del contexto de surgimiento de las Transferencias Monetarias Condicionadas en Argentina, su continuidad y los sujetos sociales a las que se orientan. En II Jornadas de Investigadores del INES CONICET-UNER. Jornadas llevadas a cabo en el Instituto de Estudios Sociales, Universidad Nacional de Entre Ríos, Paraná.

Kessler, G. (2002). La experiencia escolar fragmentada. Estudiantes y docentes en la escuela media en Buenos Aires. Buenos Aires: UNES, CO, IIPE.

Kessler, G. (2007). Juventud rural en América latina. Panorama de las investigaciones actuales en Educación, desarrollo rural y juventud. En $\mathrm{R}$. Bruniard (Ed.), La educación de los jóvenes de provincias del NEA y NOA en Argentina (pp. 16-67). Recuperado de http://unesdoc.unesco.org/images/0015/001507/150789s.pdf

Kravetz, S. (2012). La política de la igualdad educativa. Reflexiones sobre el programa provincial "Inclusión/Terminalidad de la Escuela Secundaria". Cuadernos de educación, 10(10), 1-15.

Levinson, B. (2002). Todos somos iguales: Cultura y aspiración estudiantil en una escuela secundaria mexicana. México: Santillana.

Ligorria, V. (2016). Experiencias formativas de jóvenes rurales en escuelas secundarias con albergues de Córdoba. Educación, Formación e Investigación, 2(4). Recuperado de http://ppct.caicyt.gov.ar/index.php/ efi/article/viewFile/9921/9037

Mayer, S. \& Vlasic, V (2016). Escuelas Secundarias Rurales: Estudio descriptivo de la modalidad rural de educación secundaria en Entre Ríos (Informe Final Proyecto PIDA). Paraná: Universidad Autónoma de Entre Ríos.

Mayer, S. (2014). Educación Rural, Inmigración y Relaciones Sociales. Dos procesos de colonización agrícola en la provincia de Entre Ríos. Buenos Aires: La Colmena. 
Miranda, E. (2013). De la selección a la universalización. Los desafíos de la obligatoriedad de la educación secundaria. Espacios en Blanco. Revista de Educación, 23, 9-32.

Neufeld, M. R. (2014). El campo de la antropología y la educación en la Argentina: problemáticas y contextos. En S. P. Tosta, G. Rocha \& S. Pereyra (Eds.), Diálogos sem fronteira: história, etnografía e educação em culturas ibero-americanas (pp. 51-72). Belo Horizonte: Autêntica.

Olivera Rodríguez, I. (2009). Los sentidos de la escolaridad. O la relación juventud rural - escuela frente a los procesos de exclusión. Revista Peruana de Investigación Educativa, 1(1), 61-90.

Padawer, A., Greco, J. \& Rodríguez Celín, L. (2013). Educación y territorio en el SO misionero: la escuela secundaria obligatoria en el contexto rural. Revista del IICE, 33, 47-64.

Padawer, A., Rodríguez Celín, L., Canciani, M. L, Greco, J. \& Soto, A. (2015). La obligatoriedad de la secundaria: las modalidades de educación intercultural bilingüe y educación rural en el sudoeste misionero. Boletín de Antropología y Educación, 6(9), 71-78.

Petitti, E. M., Chelotti, T. \& Schmuck, E. (2018). La educación en los campos del norte en perspectiva histórica. Una aproximación a los departamentos de La Paz, Feliciano y Federal (Entre Ríos, Argentina). En XIII Congreso Iberoamericano de Historia de la Educación. Congreso llevado a cabo en la Universidad de la República, Montevideo.

Pinkasz, D. (2015). Introducción. En Autor (Ed), La investigación sobre educación secundaria en la Argentina en la última década (pp. 5-11). Ciudad Autónoma de Buenos Aires: Flacso.

Pizarro, C. (2014). La entrevista etnográfica como práctica discursiva: análisis de caso sobre las pistas meta-discursivas y la emergencia de categorías nativas. Revista de antropología, 57(1), 461-496.

Plencovich, M., Constantini, A. \& Bocchiocchio, A. (2009). La educación agropecuaria en la Argentina. Génesis y estructura. Buenos Aires: CICCUS.

Reguillo, R. (2000). Emergencia de culturas juveniles. Estrategias del desencanto. Bogotá: Grupo editorial Norma.

Reula, F. (1969). Historia de Entre Ríos. Política, étnica, económica, social, cultural y moral (Tomo 2). Santa Fe: Castellví.

Roa, L (2017). Juventud rural y subjetividad. La vida entre el monte y la ciudad. Buenos Aires: Grupo Editor Universitario.

Rockwell, E. (2005) La apropiación, un proceso entre muchos que ocurren en ámbitos escolares. Memoria, conocimiento y utopía, 1, 28-38.

Rockwell, E. (2006). Los niños en los intersticios de la cotidianeidad escolar: ¿resistencia, apropiación o subversión? En XI Simposio Interameri- 
Jóvenes rurales en la escuela secundaria del campo

cano de Etnografía de la Educación. Simposio llevado a cabo en la Universidad Nacional de Buenos Aires, Buenos Aires.

Rockwell, E. \& Mercado, R. (1986). La escuela, lugar de trabajo docente. Descripciones y debates. México: DIE, CINVESTAV, IPN.

Rockwell, E. (2009). La experiencia etnográfica: historia y cultura en los procesos educativos. Buenos Aires: Paidós

Román, M. (2003). Los jóvenes rurales en Argentina. Elementos para una estrategia de desarrollo rural. Buenos Aires: Ministerio de la Producción, Secretaría de Agricultura, Ganadería, Pesca y Alimentos. Dirección de Desarrollo Agropecuario, PROINDER.

Román, M. (2011). Juventud en áreas rurales de Argentina. Impacto de los cambios ocurridos en la década del noventa. Buenos Aires: Universidad de Buenos Aires.

Romero Acuña, M. (2017). Contexto educativo del Delta Medio del Paraná. Una aproximación a la cotidianeidad escolar en el pasaje del primero al secundario en escuelas de isla. En Congreso ALAS. Congreso llevado a cabo en la Universidad de la República, Montevideo.

Ruiz Peyré, F. (2010). Espacios de acción, perspectivas de vida y desigualdades de género de jóvenes rurales del oeste argentino. RITA, 4. Recuperado de http://www.revue-rita.com/dossier-thema-61/ espacios-de-accion.html

Saintout, F. (2009). Jóvenes, el futuro llegó hace rato: percepciones de un tiempo de cambios: familia, escuela, trabajo y política. Buenos Aires: Prometeo Libros.

Schmuck, E (2017). Juventudes, educación secundaria y trayectorias de vida en el espacio social rural. Puntos de partida de un estudio que se realiza en las colonias $N^{\circ} 3$ y 14 del departamento La Paz (Entre Ríos). Trabajo presentado en las $1^{\circ}$ Jornadas de Investigación en Ciencias Sociales "Principales resultados y líneas de trabajo" del Instituto de Estudios Sociales, Universidad Nacional de Entre Ríos, Paraná.

Schmuck, E. (en prensa). Juventudes en plural, territorios en transformación. Hacia un estado del arte de los estudios sobre juventudes rurales en Argentina. Revista de la Pós de la UnB, 3.

Terigi, F. (2008). Los cambios en el formato de la escuela secundaria argentina: por qué son necesarios, por qué son tan difíciles. Propuesta Educativa, 29, 63-71.

Tiramonti, G. (2015). Escuela secundaria siglo XXI: un recorrido por algunas de sus reformas de cara a los desafíos de la sociedad contemporánea. Propuesta Educativa, 44(44), 6-7.

Visintini, M. E. \& Welsch, J. (2017). Distribución de la población rural en la provincia de Entre Ríos 1991-20101. En N. Finelli y M. Cardoso (Eds.), Temas de investigación y debate en la Ciencia Geográfica. Santa Fe: Editorial de la Universidad Nacional del Litoral. 


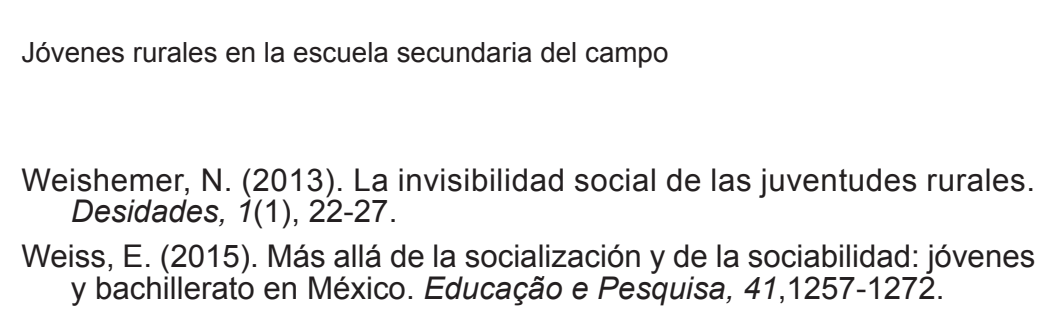

